\title{
Streamwise-localized solutions at the onset of turbulence in pipe flow
}

\author{
M. Avila ${ }^{1}$, F. Mellibovsky ${ }^{2}$, N. Roland ${ }^{3}$ \& B. Hof ${ }^{3,4}$ \\ ${ }^{1}$ Institute of Fluid Mechanics, Friedrich-Alexander-Universität Erlangen-Nürnberg, 91058 Erlangen, Germany \\ ${ }^{2}$ Castelldefels School of Telecom and Aerospace Engineering (EETAC), \\ Universitat Politècnica de Catalunya, 08860 Barcelona, Spain \\ ${ }^{3}$ Max Planck Institute for Dynamics and Self-Organization (MPIDS), 3707ry Göttingen, Germany \\ ${ }^{4}$ Institute of Science and Technology Austria, 3400 Klosterneuburg, Austria
}

(Dated: June 11, 2013)

\begin{abstract}
Although the equations governing fluid flow are well known, there are no analytical expressions that describe the complexity of turbulent motion. A recent proposition is that in analogy to low dimensional chaotic systems, turbulence is organized around unstable solutions of the governing equations which provide the building blocks of the disordered dynamics. We report the discovery of periodic solutions which just like intermittent turbulence are spatially localized and show that turbulent transients arise from one such solution branch.
\end{abstract}

PACS numbers: 47.52.+j,47.27.Cn,47.54.-r

Fluids move in a well ordered fashion (laminar flow) when their velocity is small and in this case the flow field can usually be analytically derived from the equations of motion, the Navier-Stokes equations. However, as the inherent velocity and length scales become large, turbulence sets in and most flows of practical interest are highly disordered in space and time. Landau and Hopf proposed in the forties that this transition occurs via an infinite sequence of bifurcations starting from laminar flow [1]. This route to turbulence, later shown to consist of only a few bifurcations by Ruelle and Takens [2], is a well established paradigm for transition in many systems. In flow down a straight circular pipe, however, turbulence arises despite linear stability of the laminar flow [3], and thus the former scenario is in principle inapplicable. Moreover, in pipes just like in many other wall-bounded flows turbulence first manifests itself in localized spots surrounded by laminar flow. Although experimental observations of localized turbulent structures date back to the first comprehensive investigations of turbulence [3] and their structure and kinematics have been studied extensively [4], a theoretical understanding is missing. More recent studies have shown that turbulent spots (called puffs in pipe flow) are generally of transient nature and that their decay is memoryless [5, 6]. Nevertheless turbulence eventually becomes sustained once these structures begin to proliferate and their spreading rate outweighs their decay [7]. The Reynolds number $(R e=D U / \nu$, where $D$ is the pipe diameter, $U$ the mean velocity and $\nu$ the kinematic viscosity of the fluid) at which these processes balance marks a phase transition to sustained turbulence. Despite such recent advances, how these turbulent structures arise from the equations of motion is unknown.

Numerical studies of flows in short periodic domains led to the important discovery of invariant solutions of the Navier-Stokes equations featuring the main ingredients of the self-sustaining cycle of turbulent shear flows [8]. In pipe flow, the simplest of these solutions are trav- eling waves [9], satisfying

$$
\boldsymbol{v}(x, r, \theta, t)=\boldsymbol{v}(x-c t, r, \theta),
$$

where $(x, r, \theta)$ are cylindrical coordinates, $t$ time and $c$ the wave-speed. Traveling waves are frozen as they propagate, i.e. they are relative equilibria. Although all these exact numerical solutions are unstable, and hence cannot be directly observed in experiments, the number of unstable directions is small, so it is expected that they play an important role in organizing the phase-space dynamics of turbulence [10]. As traveling waves have no dynamics but only drift in the propagation direction, more complex solutions are required to capture the properties of turbulent flows. The next level of complexity in the hierarchy of invariant solutions of the governing equations is provided by relative periodic orbits (RPOs)

$$
\boldsymbol{v}(x, r, \theta, t)=\boldsymbol{v}(x-\bar{c} T, r, \theta, t+T),
$$

for which the motion appears as $T$-periodic in a frame comoving at speed $\bar{c}$. Relative periodic orbits bifurcating from traveling waves [11] and embedded in turbulence 12. have been recently discovered in short pipes.

Although some aspects of the traveling wave solutions found in small domains, like the symmetry and the vortex streak arrangement have also been observed in turbulent pipe experiments [13], the streamwise structure is qualitatively different. While traveling waves are streamwise periodic, with a periodicity of a few $D$, all turbulent structures observed close to onset are localized. Turbulent puffs have distinct laminar-turbulent interfaces characterized by a sharp velocity change at the upstream interface and a slow adjustment downstream. In this Letter, we present the first localized solutions that contain all spatial features of turbulent puffs and show how turbulent transients emerge from them.

Numerical simulations of pipe flow were carried out using a spectral code [14 and a hybrid spectral finitedifference code [17], with excellent agreement between 


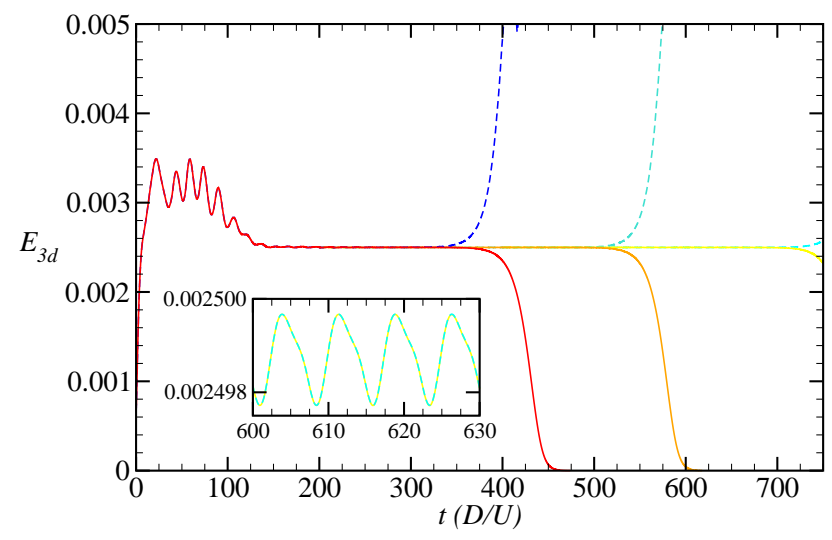

FIG. 1. Dynamics of pipe flow at the edge $(R e=2200)$. At $t=0$ a disturbance is applied to the laminar flow and the evolution of kinetic energy (of three-dimensional Fourier modes) is subsequently monitored. The dashed lines correspond to flow trajectories that shoot up to turbulence, whereas the solid lines show trajectories that relaminarize. The edgetracking algorithm is applied to obtain trajectories that hang around on the edge of chaos. The periodic oscillations shown in the inset (close up) suggest that trajectories on the edge are attracted to a RPO.

them. The computational domain was chosen to be long $(40 D)$ with periodic boundary conditions in the streamwise direction. In such long domains, just like in experiments, turbulence takes the form of localized puffs and the agreement with experiments even of very subtle features like lifetime statistics is very good [6].

At first our investigation focused on the laminarturbulent phase-space boundary by looking for initial conditions that neither turn turbulent nor relaminarise but remain in the dividing edge [15]. In long pipes the attractor in the edge (called edge state) was found to be localized but at the same time chaotic [16, 17] and the dynamics turned out to be too complex to identify underlying invariant solutions. Although approaches to nearly periodic dynamics were reported in studies of symmetric invariant subspaces, in long pipes the edge state was always found to be chaotic [18]. Here we simplified the problem by restricting the dynamics subject to a $\pi$ rotational symmetry with respect to the pipe axis

$$
[u, v, w](x, r, \theta, t)=[u, v, w](x, r, \theta+\pi, t)
$$

and the reflectional symmetry

$$
[u, v, w](x, r, \theta, t)=[u, v,-w](x, r,-\theta, t),
$$

where $u, v$ and $w$ are the axial, radial and azimuthal velocities, respectively. The reflectional symmetry (4) prohibits rotations about the pipe axis. Note that any solutions found in the subspace are necessarily also solutions of the full space and hence represent physical (symmetric) flow states.

The edge-tracking algorithm is as follows. First a localized disturbance is applied to the laminar flow [19] and if
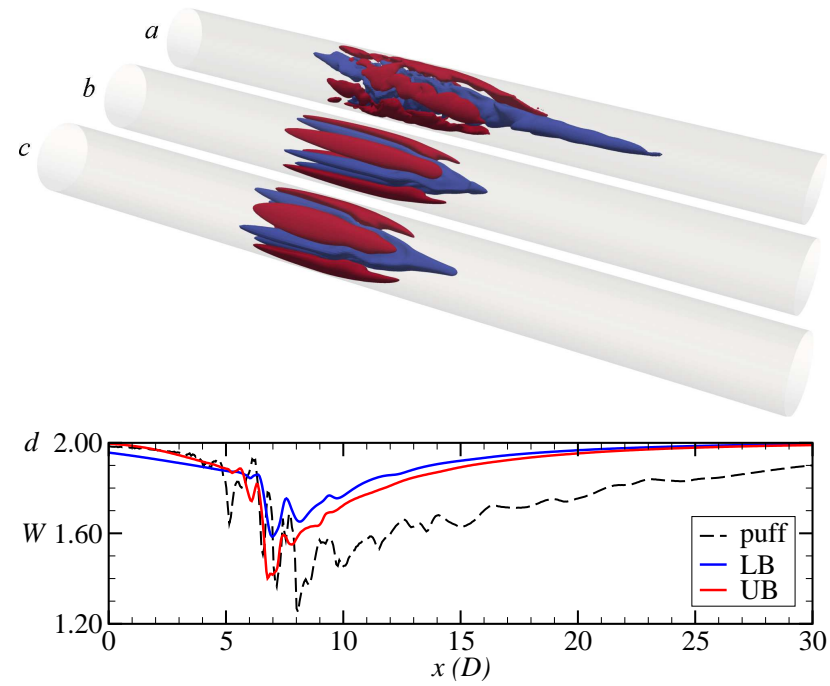

FIG. 2. (a) Turbulent puff at $R e=1900$ and reflectionsymmetric RPOs with $\pi$-rotational-symmetry at: (b) the edge (LB) at $R e=1900$ and (c) $\mathrm{UB}$ at $R e=1500$. Isosurfaces of streamwise velocity at $0.2 U$ (red) and $-0.2 U$ (blue) are shown. The laminar profile has been subtracted in all cases to highlight the three-dimensional structure of the flow and the views have been shrank by a factor of 4 in the streamwise direction. $40 \mathrm{D}$ are shown out of a simulation domain of $50 \mathrm{D}$ (puff) and $40 D$ (LB, UB). (d) Streamwise velocity along the pipe centerline for the structures in (a)-(c).

sufficiently strong it evolves into a turbulent puff. Subsequently, the amplitude of this puff, to which the laminar parabolic flow has been subtracted, is rescaled to obtain a new initial condition $\boldsymbol{v}_{\alpha}=\boldsymbol{v}_{\text {lam }}+\alpha\left(\boldsymbol{v}-\boldsymbol{v}_{\text {lam }}\right)$, where $\alpha$ is a constant $\alpha \in(0,1), \boldsymbol{v}$ the velocity field of the puff and $\boldsymbol{v}_{\text {lam }}$ the laminar flow. A simple bisection algorithm is then used to find the value of $\alpha$ for which the temporal evolution of $\boldsymbol{v}_{\alpha}$ neither relaminarizes nor goes to turbulence but remains on the edge. The procedure is illustrated in figure 1 at $R e=2200$. After an initial transient the temporal evolution rapidly relaxes onto a periodic oscillation, suggesting that the edge state is a RPO. Note that as time evolves new refinement bisection iterations have to be applied to keep the trajectory on the edge.

A snapshot of the edge velocity field was fed as initial guess into a purposely designed Newton-Krylov solver based on the time-stepping code 14 using standard techniques [11, 20] and rapidly converged to a RPO with pe$\operatorname{riod} T=15.0 \mathrm{D} / U$ and average drift speed $\bar{c}=1.52 \mathrm{U}$. Note that in order to achieve convergence we require that the residual $r=\|\boldsymbol{v}(T)-\boldsymbol{v}(0)\|<10^{-10}\|\boldsymbol{v}(0)\|$, where the velocity field $\boldsymbol{v}(T)$ has been appropriately shifted to account for drift. Figure 1 shows that the energy oscillations have a period of $T / 2$. This is due to a spatiotemporal symmetry possessed by this solution: at $t=T / 2$ the velocity field is the same as $t=0$ but reflected with respect to the plane at $\theta=45^{\circ}$ (note that the plane of im- 
posed reflection-symmetry is at $\theta=0$ ). Figure $2 \mathrm{~b}$ shows a snapshot of the RPO. The similarity in the topology of its low and high velocity streaks with those of a turbulent puff (shown in 2a) is remarkable. A close inspection of the topology of streaks and vortices of this solution points at a possible connection with a stream-wise periodic traveling wave [21] (D2). We found that at $R e=2200$ this traveling wave is the edge state in short pipes of length $\lambda \lesssim 5 D$, whereas in the range $5 \lesssim \lambda \lesssim 10 D$ the edge state is chaotic. Although a localized RPO is obtained as long as $\lambda \gtrsim 10 D$, for $\lambda \lesssim 15 D$ the periodic boundary conditions ostensibly interfere with localization. For the pipe length $\lambda=40 D$ used in the results presented here, the periodic boundary conditions have no longer an effect on localization (which was tested by repeating some simulations for $\lambda=80 D$ ).

As the Reynolds number is reduced the localized RPO (henceforth referred to as LB, which stands for lower branch solution) keeps fulfilling its role of separating trajectories that relaminarize from those that increase in energy towards turbulence. For $R e<1530$ trajectories above the edge no longer result in turbulent transients but approach instead a stable (within the $\pi$-rotationaland reflection-symmetric space) localized RPO. The visualisation of this new solution (hereafter UB, standing for upper branch solution) is shown in figure 2 c and reveals a striking structural resemblance to turbulent puffs. As pointed out above, a typical signature of puffs is the sharp transition from laminar to turbulent flow at the trailing interface followed by a slow recovery towards the laminar velocity along its diffuse leading interface (see the black curve in figure 22 $\mathrm{d}$ ). This landmark of puffs is shared by LB (red curve) and UB (blue curve) and further demonstrates that the properties of localized turbulence can be captured by exact numerical solutions of the Navier-Stokes equations.

At $R e \approx 1430$ UB merges with LB at a saddle-node bifurcation (see figure $3 \mathrm{k}$ ) and below this bifurcation no dynamics other than laminar are found. By continuing the UB towards larger Reynolds number we could identify a bifurcation cascade leading to turbulent transients. At $R e \approx 1530$ the UB undergoes a Neimark-Sacker bifurcation leading to a stable 2-torus that breaks up into chaos at $R e \approx 1540$. Although at the onset of chaos the attractor explores only a small portion of the phase space, this portion grows explosively as $R e$ is increased and the chaotic attractor appears to collide with LB at $R e \approx 1545$. This boundary crisis is likely related to the appearance of a homoclinic tangle on the edge [22]. Beyond this point the attractor becomes leaky: trajectories can relaminarize after long transients. Following the ensuing chaotic saddle to larger Re confirms that turbulence in the subspace originates at this bifurcation, as illustrated in the phase-space portrait of figure $3 \mathrm{~b}$. We note that similar bifurcation scenarios but starting from relative equilibria have been observed in short pipes 23]
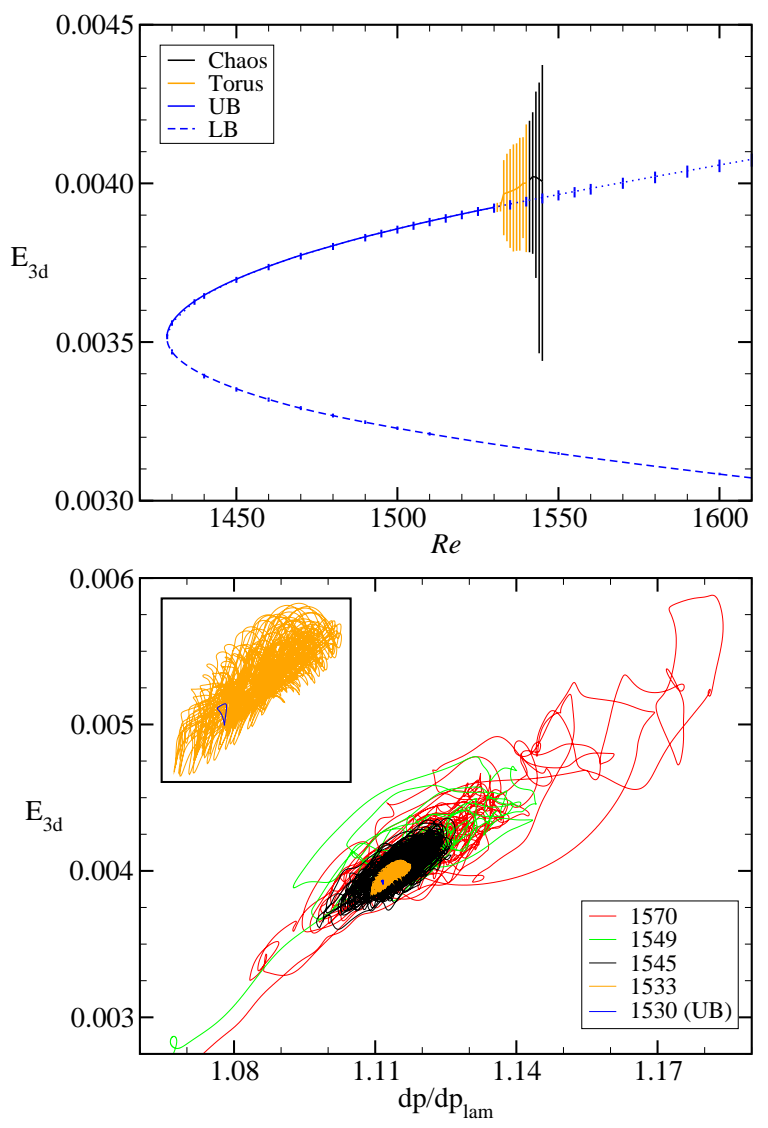

FIG. 3. (a) A saddle-node bifurcation gives rise to localized RPOs at $R e \approx 1430$ : UB is stable up to $R e \approx 1530$, where it undergoes a supercritical Neimark-Sacker bifurcation leading to a relative 2-torus. Subsequently the torus breaks up to chaos at $R e \approx 1540$ and the chaos becomes transient at $R e \approx$ 1545. The bars show the variation of energy over a period (Newton-converged $\mathrm{LB}$ and $\mathrm{UB}$ ) and over long runs (torus and chaos). LB has a single unstable direction and is the edge state. (b) Phase-portrait of the dynamics at several Re projected onto a two-dimensional plane defined by the energy (of three-dimensional Fourier modes) and pressure gradient required to drive a constant flow rate, normalized with the pressure gradient of laminar flow. The inset is a close up showing the UB and torus.

and in small plane Couette cells 24], thus lacking the spatial complexity and laminar turbulent interfaces observed in practice. In these small cells a chaotic attractor emerges via period doubling bifurcations and subsequently leads to transients [24].

The robustness of the RPOs and transition scenario were tested with respect to spatial resolution and timestep $\delta t$. We used $\delta t=0.0025 D / U$ and $K= \pm 320$ axial Fourier modes, $M= \pm 16$ azimuthal Fourier modes (for $\theta \in[0, \pi])$ and $N=40$ points in the radial direction. With these values the solutions are well converged and the bifurcation points are accurate to better than $0.5 \%$. For lower resolutions the bifurcations are shifted towards lower $R e$, whereas the opposite effect is observed by in- 
creasing $\delta t$. Nevertheless, the scenario remains qualitatively unchanged. Note that in the full space the solutions found here have several additional instabilities and hence cannot be computed by edge tracking and time-stepping. We performed several simulations starting from them but dropping the symmetry restrictions and observed similar transients.

In summary, we have discovered exact numerical solutions of the Navier-Stokes equations that share structure and spatial complexity with turbulence at onset. We have furthermore shown that a bifurcation sequence is responsible for giving rise to transient turbulence. In contrast to the classical Ruelle-Takens model, in pipe flow chaotic motion arises locally originating from the discovered localized solutions. This is a key difference to the much simpler transition scenarios in linearly unstable flows, such as Rayleigh-Bénard convection [25] and Taylor-Couette flow [26], where the bifurcation sequence starts from the base flow and instability occurs globally in space. Localized solutions can therefore be regarded as the nuclei of disordered motion in linearly stable shear flows. It is likely that in full space chaotic dynamics simultaneously arises from distinct nuclei and that the corresponding repellers merge in global bifurcations as $R e$ grows, increasing the complexity of the turbulent transients. One of the outstanding challenges towards an understanding of the spatiotemporal complexity encountered in shear flows close to onset is the identification of the mechanism leading to spatial localization [27].

We thank A. P. Willis for sharing his code. The research leading to these results has received funding from the Max Planck Society and the European Research Council under the European Union's Seventh Framework Programme (FP/2007-2013) / ERC Grant Agreement 306589. We acknowledge computing resources from GWDG and the Jülich Supercomputing Centre (grant HGU16).

[1] L. D. Landau, CR Acad. Sci. URSS 44, 1 (1944); E. Hopf, Communications on Pure and Applied Mathematics 1, 303 (1948).

[2] D. Ruelle and F. Takens, Commun. Math. Phys. 20, 167 (1971); L. D. Landau and E. Lifshitz, Fluid Mechanics, 2nd ed., Course of Theoretical Physics, Vol. 6 (Pergamon, 1987).

[3] O. Reynolds, Proc. Roy. Soc. London 35, 84 (1883).

[4] I. J. Wygnanski and F. H. Champagne, J. Fluid Mech. 59, 281 (1973); I. J. Wygnanski, M. Sokolov, and D. Friedman, ibid. 69, 283 (1975); P. R. Bandyopadhyay, ibid. 163, 439 (1986); M. Nishi, B. Ünsal, F. Durst, and G. Biswas, ibid. 614, 425 (2008); M. Shimizu and S. Kida, Fluid Dynamics Research 41, 045501 (2009);
C. van Doorne and J. Westerweel, Phil. Trans. Roy. Soc. A 367, 489 (2009); B. Hof, A. De Lozar, M. Avila, X. Tu, and T. Schneider, Science 327, 1491 (2010).

[5] B. Hof, J. Westerweel, T. Schneider, and B. Eckhardt, Nature 443, 59 (2006); B. Hof, A. De Lozar, D. Kuik, and J. Westerweel, Phys. Rev. Lett. 101, 214501 (2008).

[6] M. Avila, A. Willis, and B. Hof, J. Fluid Mech. 646, 127 (2010).

[7] K. Avila, D. Moxey, A. De Lozar, M. Avila, D. Barkley, and B. Hof, Science 333, 192 (2011).

[8] M. Nagata, J. Fluid Mech. 217, 519 (1990); F. Waleffe, Phys. Rev. Lett. 81, 4140 (1998).

[9] H. Faisst and B. Eckhardt, Phys. Rev. Lett. 91, 224502 (2003); H. Wedin and R. R. Kerswell, J. Fluid Mech. 508, 333 (2004).

[10] R. R. Kerswell, Nonlinearity 18, R17 (2005); B. Eckhardt, T. M. Schneider, B. Hof, and J. Westerweel, Ann. Rev. Fluid Mech. 39, 447 (2007) J. Gibson, J. Halcrow, and P. Cvitanović, J. Fluid Mech. 611, 107 (2008); G. Kawahara, M. Uhlmann, and L. van Veen, Ann. Rev. Fluid Mech. 44, 203 (2012).

[11] Y. Duguet, C. C. T. Pringle, and R. R. Kerswell, Phys. Fluids 20, 114102 (2008)

[12] A. Willis, P. Cvitanović, and M. Avila, J. Fluid Mech. 721, 514 (2013).

[13] B. Hof, C. W. H. van Doorne, J. Westerweel, F. Nieuwstadt, H. Faisst, B. Eckhardt, H. Wedin, R. R. Kerswell, and F. Waleffe, Science 305, 1594 (2004); B. Hof, C. W. H. van Doorne, J. Westerweel, and F. T. M. Nieuwstadt, Phys. Rev. Lett. 95, 214502 (2005); A. de Lozar, F. Mellibovsky, M. Avila, and B. Hof, Phys. Rev. Lett. 108, 214502 (2012).

[14] A. Meseguer and F. Mellibovsky, Appl. Num. Math. 57, 920 (2007).

[15] T. M. Schneider, B. Eckhardt, and J. A. Yorke, Phy. Rev. Lett. 99, 034502 (2007).

[16] F. Mellibovsky, A. Meseguer, T. M. Schneider, and B. Eckhardt, Phys. Rev. Lett. 103, 054502 (2009).

[17] A. P. Willis and R. R. Kerswell, J. Fluid Mech. 619, 213 (2009)

[18] Y. Duguet, A. P. Willis, and R. R. Kerswell, J. Fluid Mech. 663, 180 (2010).

[19] F. Mellibovsky and A. Meseguer, Phys. Fluids 19, 044102 (2007).

[20] D. Viswanath, J. Fluid Mech. 580, 339 (2006).

[21] Y. Duguet, A. P. Willis, and R. R. Kerswell, J. Fluid Mech. 613, 255 (2008).

[22] L. van Veen and G. Kawahara, Phys. Rev. Lett. 107, 114501 (2011); N. R. Lebovitz, Communications in Nonlinear Science and Numerical Simulation 17, 2095 (2012).

[23] F. Mellibovsky and B. Eckhardt, J. Fluid Mech. 709, 149 (2012).

[24] T. Kreilos and B. Eckhardt, Chaos: An Interdisciplinary Journal of Nonlinear Science 22, 047505 (2012).

[25] A. Libchaber, C. Laroche, and S. Fauve, Journal de Physique Lettres 43, 211 (1982).

[26] J. P. Gollub and H. L. Swinney, Phys. Rev. Lett. 35, 927 (1975).

[27] E. Knobloch, Nonlinearity 21, T45 (2008); T. M. Schneider, J. F. Gibson, and J. Burke, Phys. Rev. Lett. 104, 104501 (2010). 